\title{
Les nécrologies des Poilus dans les journaux de tranchées de la Grande Guerre
}

Loredana Trovato $^{1}$

The purpose of this article is to contribute to the analysis of a genre, 'discourse about the dead', which has not been studied very much from the standpoints of rhetorical argumentation and discourse analysis. In particular, I shall focus on the obituaries published in the newspapers of the French trenches of World War I in an attempt to highlight their main characteristics in connection with the processes of constructing biographical memory. After presenting the corpus, I shall tackle the question of the writer-reader relationship and classify the obituaries into three specific types. Finally, I shall examine the particularities of these three categories and the ways in which these obituaries tell the story of each soldier's life and death.

Cet article propose de contribuer à l'analyse d'un genre, 'le discours sur les morts', qui a été relativement peu étudié sous l'angle de l'argumentation rhétorique et de l'analyse du discours. Je me focaliserai en particulier sur les nécrologies parues dans les journaux de tranchées français de la Première Guerre mondiale, dont j'essaierai de mettre en relief les caractéristiques principales en rapport avec les processus de construction de la mémoire biographique. Après la présentation du corpus utilisé, j'aborderai la question du lien scripteur / lecteur et je classerai les nécrologies en trois types distincts dont j'examinerai ensuite les spécificités et les modalités constituant le récit de la vie et de la mort du soldat.

\section{Liminaire}

Le sujet de ce numéro offre un terrain fertile à l'analyse du genre de la nécrologie qui fournit des pistes intéressantes et utiles pour investiguer les mécanismes par lesquels se construit la mémoire biographique.

Dans cet article, je m'occuperai spécifiquement des textes qu'on rassemble sous l'étiquette de 'discours sur les morts', publiés dans les journaux de tranchées français de la Grande Guerre. Envisagés en tant que narrations laconiques de la vie des soldats décédés au cours des combats, ils présentent une dimension non seulement informative et émotionnelle mais également éthique et argumentative car ils établissent le topos du Poilu, vu comme un modèle d'héroïque abnégation et de dévouement absolu à la patrie. Après une courte introduction à mon corpus, je me

\footnotetext{
${ }^{1}$ Université d'Enna « Kore », Italie.
} 
pencherai sur leur architecture textuelle ainsi que sur leurs modes de circulation et leur contribution à la définition de l'ethos individuel et collectif ${ }^{2}$. Je me concentrerai sur les caractéristiques formelles mises en œuvre pour raconter l'évènement tragique et les derniers moments du Poilu «qui a fait le sacrifice de sa vie» pour sauver la France de «l'envahisseur allemand ${ }^{3}$. J'essaierai en outre de dégager les modalités intervenant dans la construction de la biographie du défunt, ce qui permettra enfin de relever comment la plupart de ces nécrologies obéissent au prototype de l'exemplum, c'est-à-dire - d'après la définition de Marc Angenot - d'un " micro-récit [...] articulé à une éthopée, au portrait d'un individu en situation, le tout supposé être suffisamment frappant parce que 'typique' ${ }^{4}$.

\section{Les journaux de tranchées et les discours sur les morts}

La Première Guerre mondiale a favorisé le développement d'une riche littérature, ainsi que la production d'un vaste ensemble de «discours sur les morts », expression utilisée pour désigner les «lieux de mémoire»- selon l'acception de Pierre Nora ${ }^{5}$ - que sont les productions textuelles concernant l'annonce de la mort individuelle sous leurs formes très diverses d'oraisons et éloges funèbres, d'avis et de faire-part de décès.

Leur teneur, force illocutoire et dimension constitutive se manifestent à travers leur emplacement (spatial et temporel), ou positionnement «dans le système des relations dans lequel $»^{6}$ ils sont pris, à savoir les journaux de tranchées, un ensemble très vaste de bulletins et canards de guerre, écrits à la main ou imprimés par les soldats eux-mêmes pendant la période 1914-187 . Il

\footnotetext{
2 Pour approfondir ces concepts et leurs implications au niveau de l'argumentation rhétorique, cf. Ruth AMOSSY, La Présentation de soi. Ethos et identité verbale, Paris, PUF, 2010.

${ }^{3}$ Les expressions citées sont des formules typiques qu'on peut trouver partout dans les nécrologies des journaux de tranchées.

${ }^{4}$ Marc ANGENOT, «L'exemplum dans l'idéologie, ou: l'Affaire Dreyfus avant 1894 », in Emmanuelle DANBLON, Victor FERRY, Loïc NICOLAS, Benoît SANS (dir.), Rhétoriques de l'exemple. Fonctions et pratiques, Besançon, Presses Universitaires de Franche-Comté, 2014, p. 39.

${ }^{5}$ Pierre NORA, Les Lieux de mémoire. T.1. La République, Paris, Gallimard, 1984.

${ }^{6}$ Pascale DELORMAS, De l'autobiographie à la mise en scène de soi. Le cas Rousseau, Limoges, LambertLucas, 2012, p. 17.

${ }^{7}$ Sur la base de quelques enquêtes publiées après le conflit, on a repéré environ 474 publications. Ce chiffre est attesté dans le livre de Jean-Pascal SOUDAGNE, Le Quotidien des soldats dans les tranchées (Saint-Cloud, 1418 éditions, 2009, p. 128) à la suite d'André CHARPENTIER (Feuilles bleu horizon. Le livre d'or des journaux
} 
s'agit de l'une des nouveautés remarquables de cette guerre, qui se veut comme la première à dimension médiatique. Les soldats les utilisaient pour communiquer, transmettre des dépêches, mais surtout pour se distraire et chasser le « cafard $»^{8}$, comme l'attestent à maintes reprises titres (par exemple, " Gardons le sourire »), sous-titres (par exemple, " Organe hilarant ») et préfaces programmatiques ${ }^{9}$. Néanmoins, ils ne peuvent s'empêcher de raconter la mort aussi, parce que, comme une sorte d'impératif catégorique, il faut parler des camarades qui sont tombés au champ d'honneur, il faut les mythifier, faire leur apologie, en relatant leur brève existence et leur meurtre atroce. De cette manière, ils contribuent à la création de l'image légendaire du Poilu : le soldat qui s'est immolé pour la nation n'est pas un Poilu quelconque, car c'est LE Poilu, un modèle d' « exceptionnelles vertus », «d'inestimable courage », du véritable « esprit gaulois », à opposer à l'ennemi, au « terrible et redoutable Boche $»^{10}$.

Sur le plan structurel, ces nécrologies se caractérisent par une sorte d'hétérogénéité fonctionnelle et formelle. J'en ai repéré trois types principaux qui présentent des traits distinctifs : (a) les nécrologies de type $1[\mathrm{~N} 1]$ sont à peu près des «phrases sans texte ${ }^{11}$, des énoncés détachés, des euphorisations qui ne correspondent souvent qu'à des groupes nominaux; (b) Les nécrologies de type 2 [N2] sont constituées d'avis et de faire-part de décès à la visée notamment informative et à l'organisation des contenus assez régulière (données personnelles du soldat, lieu, date, âge de mort, condoléances adressées à la famille) ; (c) enfin, les nécrologies de type 3 [N3] sont des narrations plus longues qui adoptent généralement le style de l'oraison et de l'éloge funèbre et qui peuvent être rapprochées du genre épidictique.

Étant donné les objectifs de cet article, je m'intéresserai uniquement aux nécrologies de type 2 et 3 , dans lesquelles il sera possible de mettre en lumière les caractéristiques du récit de la vie et

\footnotetext{
du front, 1914-1918, Triel-sur-Seine, Éd. Italiques, 2007 [1935]) et Georges THURIOT-FRANCHI (Les Journaux de tranchées, Paris, Nevers, 1921).

${ }^{8}$ Synonyme de «ennui, mélancolie, idées noires », selon François DECHELETTE in L'Argot des Poilus. Dictionnaire humoristique et philologique du langage des soldats de la grande guerre de 1914, Paris, Jouve \& $\mathrm{C}^{\mathrm{ie}}$ Éditeurs, 1918, p. 53.

${ }^{9} \mathrm{Cf}$. à ce propos, Loredana TROVATO, Au commencement était la tranchée. Le rôle des textes préliminaires des journaux de tranchées dans la construction / divulgation de l'ethos du combattant, in « Studii şi cercetări filologice. Seria limbi romanice », $n^{\circ} 16$, vol. 1, 2014, pp. 88-101.

${ }^{10}$ Autres formules utilisées à plusieurs reprises dans les nécrologies et dans toute la presse du front pour qualifier les combattants français.

${ }^{11}$ Dominique MAINGUENEAU, Les Phrases sans texte, Paris, A. Colin, 2012.
} 
de la fin du combattant ainsi que les modalités qui rendent compte de sa mémoire pour essayer de l'inscrire dans l'histoire.

\section{Le rapport avec le destinataire dans les nécrologies}

La question de la relation entre les deux partenaires du processus communicatif - l'émetteur et le destinataire - et de ceux-ci au sujet du message, c'est-à-dire le défunt, est essentielle du fait qu' « on parle toujours pour et en fonction de quelqu'un $»^{12}$, ce qui détermine l'exigence d'adapter constamment le discours à l'auditoire ${ }^{13}$. Dans le cas des nécrologies des journaux de tranchées, il faut, en outre, se demander si l'on peut « dire la mort de l'autre sans que cette parole varie considérablement en fonction du locuteur $»^{14}$ et de sa participation émotive au deuil. En réalité, ces nécrologies ne disent pas la mort de l'autre, parce que cet 'autre', c'est le camarade, l'ami, le frère d'armes tombé sous le feu ennemi : il n'y a pas de véritable distance affective, plutôt une profonde douleur et un grand respect, qui provoquent forcément une réponse exaltée au thème de la disparition prématurée de la jeunesse ${ }^{15}$ engagée au front. À cet égard Michel Meyer apporte ici une contribution fondamentale en ce qu'elle définit bien le rôle de la morale dans l'établissement et la rupture des liens affectifs entre les êtres humains : "plus la distance est faible entre les êtres, plus la réponse qu'ils suscitent est passionnelle. Cela se retrouve dans l'attitude morale à leur égard, où des notions plus viscérales comme le plaisir, la tristesse, la colère et la joie viennent colorer le jugement qu'on a sur eux ou qu'ils ont sur eux-mêmes et qui se prétend objectif $»^{16}$.

Qui plus est, il faut constater que le rapport émetteur / destinataire est unilinéaire et égalitaire, car les soldats sont, de façon autoréférentielle, les producteurs-récepteurs de leurs textes. Les journaux circulent de tranchée en tranchée pour n'arriver qu'en un deuxième moment à l'arrière : cela veut dire que l'émetteur, le sujet du message et le destinataire partagent le même

\footnotetext{
${ }^{12}$ Ruth AMOSSY, L'Argumentation dans le discours, Paris, A. Colin, 2013, p. 50.

${ }^{13} \mathrm{~J}$ 'emploie ce terme selon la perspective offerte à partir de la Nouvelle rhétorique, inaugurée par Chaïm Perelman et Lucie Olbrechts-Tyteca.

${ }^{14}$ Gérard JACQUIN, «Avant-propos », in id. (sous la direction de), Le Récit de la mort. Ecriture et histoire, Rennes, PUR, 2003, p. 9.

${ }^{15}$ Thème qui constitue un topos dès l'Antiquité. Il suffit de penser au célèbre fragment de Ménandre, « ôv oi $\theta \varepsilon o i ̀$

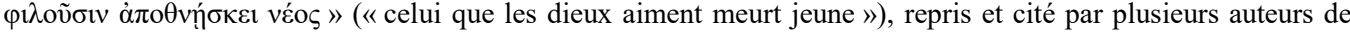
toutes les époques.

${ }^{16}$ Michel MEYER, Principia moralia, Paris, Fayard, 2013, p. 58.
} 
statut, celui de soldat. Nous avons ainsi un auditoire «homogène »: «l'orateur s'adresse à un public qui partage des valeurs, voire des objectifs, identiques $»{ }^{17}$. Par conséquent, l'orateur ne doit pas «s'adapter à l'auditoire » comme «condition sine qua non» de son efficacité discur$\operatorname{sive}^{18}$ : il doit seulement renforcer l'adhésion du public à son éthos par la négociation rhétorique, ergo le pathos, s'exprimant à l'aide de schèmes compositionnels précis et de figures telles que l'amplification et l'hyperbole.

\section{Les nécrologies de type 2}

À la différence des N3, les N2 comportent plus de contraintes déterminées par leur publication dans la rubrique des nécrologes, dont l'espace est ordinairement restreint à une demi-page : cela implique la nécessité de résumer en peu de lignes les données concernant le défunt et les circonstances de son trépas, pour terminer avec les formules (assez) stéréotypées de l'expression du deuil.

En effet, elles sont construites à partir d'un modèle figé qui correspond à peu près à celui des faire-part de décès contemporains ${ }^{19}$. Si l'on adapte le tableau proposé par Françoise Hammer, on peut distinguer trois classes d'informations caractérisant chaque N2: (a) les informations primaires se composant de l'annonce et d'une présentation rapide du disparu ; (b) des informations secondaires (facultatives) sur la date et le lieu de la cérémonie ${ }^{20} ;(c)$ des informations tertiaires (facultatives elles aussi), contenant des épigraphes, des symboles, ou les condoléances à la famille ${ }^{21}$. Voici quelques exemples :

\footnotetext{
${ }^{17}$ R. AMOSSY, L'Argumentation dans le discours, op. cit., p. 63.

${ }^{18}$ Ibid., p. 53.

19 «Texte d'information, le faire-part présente la structure stéréotypée d'un formulaire mis en place pour résoudre les problèmes rédactionnels d'un acte langagier récurrent $[\ldots]$ et réutilisable par adjonction ou substitution d'éléments circonstanciels » (François HAMMER, «Le faire-part de décès et la confrontation avec la mort », in Questions de communication, 19, 2011, p. 57).

${ }^{20}$ Ce type d'informations est, pour la plupart, absent des nécrologies des journaux de tranchées, car il était très difficile de donner de dignes sépultures aux corps des soldats morts au cours d'une bataille.

${ }^{21}$ Cf. F. HAMMER, « Le faire-part de décès et la confrontation avec la mort », op. cit., p. 57.
} 
[1] Notre ami Hervieu est décédé dans un hôpital de Salonique. Brave garçon, très ingénieux et doté d'une force herculéenne, il était, il y a deux mois, loin de nous laisser entrevoir le malheur qui vient de frapper sa famille. Encore un qui, par son humble mort travaille au salut du pays ${ }^{22}$.

[2] Une bien triste nouvelle vient de nous parvenir : Alfred Bonvous, l'Angevin, dit Coeur-deFrance, vient de trouver la mort devant Verdun. La Revue perd en lui un collaborateur dévoué. Le Compagnonnage perd une des jeunes intelligences qui auraient collaboré puissamment à l'œuvre de régénération nécessaire que poursuivent tous ceux qui aiment notre Société. A sa famille éplorée, à son oncle Auguste Bonvous, Secrétaire Central des Groupes Fraternels, nous adressons l'expression de nos plus profonds regrets et de nos condoléances émues. La rédaction ${ }^{23}$.

[3] ÉDOUARD FRINOT. Dans un accident d'atterrissage survenu le 11 juin à Berck-Plage, notre excellent camarade Frinot, qui faisait partie du centre d'aviation de Crotoy, a trouvé une mort stupide. Notre ami faisait partie depuis longtemps de cette valeureuse phalange de journalistes lorrains qui, après avoir si ardemment défendu leur patrie par d'admirables campagnes de presse, étaient accourus simplement, en août 1914, mettre leur jeunesse et leurs fortes qualités au service du pays. Frinot était venu quelquefois nous serrer la main à Paris, depuis son adhésion à notre association. Son abord sympathique nous avait immédiatement conquis. Une mort brutale nous l'enlève comme elle nous a déjà ravi beaucoup de bons amis. Que sa veuve et ses deux petites fillettes veuillent bien trouver ici, en atténuation de leur immense chagrin, l'expression de nos condoléances émues. LE COMITE ${ }^{24}$.

Les trois exemples proposés offrent une certaine idée des typologies de N2 repérées dans les journaux de tranchées : tous les textes du genre [1] donnent des indications générales sur le décès et le défunt, dont les qualités sont souvent synthétisées en une seule phrase, où abondent les adjectifs «affectivo-axiologiques » ${ }^{25}$ («brave », « très ingénieux », «doté d'une force herculéenne »), tandis que ceux du genre [2] contiennent les informations primaires et tertiaires.

${ }^{22}$ Le Cri des ravins, 15/10/1915, p. 3.

${ }^{23}$ Revue des Groupes Fraternels. Compagnons du devoir du Tour de France sous les drapeaux, Grande Guerre 1914-1918, n 12, août 1916, p. 94.

${ }^{24}$ Bulletin mensuel des journalistes professionnels mobilisés, première année, $\mathrm{n}^{\circ} 1$, juin 1917.

${ }^{25}$ Catherine Kerbrat-Orecchioni souligne que ce type d'adjectifs servent à énoncer « un jugement de valeur, et un engagement émotionnel du locuteur vis-à-vis de l'objet dénoté » (L'Énonciation, Paris, A. Colin, 2002, p. 80). 
D'Alfred Bonvous on apprend notamment ses origines, son appellatif, le lieu de sa mort et son âge (« jeunes intelligences »).

Dans les N2 plus longues, il est possible d'observer une construction rhétorique plus marquée, finalisée à l'annonce du décès et, à la fois, à la mise en valeur de l'ethos combattant, comme dans [3], où l'adhésion de l'énonciateur et du disparu aux valeurs patriotiques est confirmée par l'emploi de la première personne du pluriel, 'nous', qui sert d'indice révélateur de l'identité collective ${ }^{26}$ et de la camaraderie entre les Poilus. Ce n'est pas un hasard si Édouard Frinot est qualifié comme «notre excellent camarade » et «notre ami », faisant partie d'un groupe de journalistes-soldats, dont la valeur et la hardiesse sont mises en relief grâce à la suite d'adjectifs et d'adverbes: "valeureuse phalange », «ardemment défendu», " admirables campagnes de presse », «fortes qualités »; suite renforcée par la fausse modestie exprimée par l'adverbe « simplement », désignant leur enrôlement volontaire.

Par leur nature et emplacement, les N2 ne relatent pas un passé trop éloigné, car elles doivent rendre compte de l'actualité : c'est pourquoi on peut constater l'utilisation régulière de la construction périphrastique 'venir de + infinitif', où la distance chronologique entre l'événement tragique et le centre déictique est brève, ainsi que des temps verbaux du passé, comme le passé récent pour l'annonce et les condoléances ${ }^{27}$, le passé composé pour le récit de la mort, l'imparfait pour la biographie du soldat.

La reprise de formes et formules pour annoncer la mort «traduit non seulement les conventions communautaires, mais aussi l'acceptation de ces normes par l'énonciateur actualisant $»^{28}$. Le répertoire phraséologique qui apparaît dans ces écrits fournit en outre un indice des normes et des valeurs partagées par tous les Poilus : la répétition du mot « ami » qualifie le rapport entre le scripteur et le défunt et témoigne de l'expression sincère de la douleur pour la perte tragique. À noter ensuite le lexème «mort », qui n'est pas omis ou évité comme dans les faire-part de décès contemporains où «l'énonciateur a recours à des techniques de minoration axiologique, euphémisation ou disphémisation $»^{29}$ pour essayer de ne pas employer un mot considéré comme tabou. Ici, par contre, on met en évidence son inéluctabilité comme condition nécessaire à la valorisation et à la réputation éternelle du soldat.

${ }^{26}$ Cf. à ce sujet, le chapitre six de R. AMOSSY La Présentation de soi. Ethos et identité verbale, op. cit., pp. 156182.

${ }^{27}$ Dans le corpus analysé, on trouve le présent de l'indicatif aussi fréquent que le passé récent.

${ }^{28}$ F. HAMMER, « Le faire-part de décès et la confrontation avec la mort », op. cit., p. 57.

${ }^{29}$ Ibid., p. 58. 


\section{Les nécrologies de type 3}

Par rapport aux N2, les N3 jouissent d'une autonomie majeure et de moins de contraintes typographiques. Et pourtant, elles ne dépassent jamais la limite de deux colonnes, étant donné qu'elles sont encadrées par le plan rigide d'un journal ${ }^{30}$. En fait, il ne s'agit pas de véritables nécrologies mais d'éloges funèbres ou de portraits, qui peuvent éventuellement $\mathrm{e}^{\wedge}$ tre utilisés comme faire-part de décès.

Ces textes disposent d'une structure très proche, pour ses finalités, de celle de l'exemplum médiéval, car où on raconte la vie d'un homme quelconque qui, s'engageant au front pour défendre la France, n'a pas peur de mourir et n'hésite pas à faire le sacrifice de sa vie. Son décès le rend enfin extraordinaire, et le transforme en un modèle à suivre pour jamais.

Façonnés sur le pattern des oraisons funèbres anciennes, ils poursuivent l'objectif premier d'emporter l'adhésion à l'ethos combattant par le renforcement du pathos : à travers la vive manifestation de la douleur, ils essaient de présenter au lecteur un modèle de vertu en consignant les actions d'un homme qui a vécu et qui s'est éteint dignement. Le soldat tombé au champ d'honneur servira d'exemple pour la postérité et vivra éternellement dans la mémoire collective. Je peux citer à ce propos la conclusion de la nécrologie de Frédéric Charpin, dans laquelle l'auteur s'exclame que son sacrifice rendra son nom immortel : « Ce nom sera inscrit en lettres d'or sur une autre pierre, celle où nos enfants liront le nom glorieux des jeunes gens morts pour la patrie $»^{31}$.

Sur le plan de l'écriture, les N3 n'obéissent qu'au seul impératif rhétorique et font appel aux procédés pathémiques, à savoir l'amplification, la répétition, l'apostrophe, la périphrase, jusqu'à exploiter - en quelques cas - les topoi du panégyrique. Relevant du genre épidictique, elles se rapprochent du discours de circonstance qui permet «de renforcer les valeurs auxquelles la communauté adhère $»^{32}$. La seule différence réside dans le fait que l'auditoire ne doit pas « juger de la beauté ou de la laideur des vertus ou des vices qui sont exposés par l'oratoire ${ }^{33}$ car le jugement relatif à beauté des vertus du défunt est unanime et il n'y a pas de véritable écart entre les valeurs proposées par le scripteur et celles partagées par le lecteur. Voilà pourquoi, en ce cas, c'est la dimension esthétique qui occupe une place très importante : comme le soutient Emma-

\footnotetext{
${ }^{30}$ Il faut préciser que la plupart des journaux de tranchées ne se composaient que de quatre pages.

${ }^{31}$ Bulletin des écrivains de 1914-1915-1916, n 16, février 1916, p. 1.

${ }^{32}$ Emmanuelle DANBLON, La Fonction persuasive, Paris, A. Colin, 2005, p. 37.

${ }^{33}$ Ibidem.
} 
nuelle Danblon, l'orateur «utilisera [...] autant de figures de rhétorique que son talent l'autorisera à le faire, ce qui confère à ce type de discours plus qu'à tout autre, un statut de spectacle $»^{34}$.

Dans beaucoup de N3, on assiste en effet à une sorte de spectacularisation de la mort du brave soldat : le récit est tellement minutieux que le lecteur paraît voir la scène. La nécrologie de Pierre Baudry est, de ce point de vue, très significative :

Pierre est mort le 29 mai vers 5 heures moins un quart. Le 27 nous avions été alertés au milieu de la nuit dans les creutes du mont de Guny, où nous étions en réserve, redescendus des tranchées depuis un ou deux jours. L'attaque allemande s'était déclenchée sur notre droite. Le lendemain soir nous allions prendre position face à l'est, en avant de la ferme de Mareuil ; marche pénible, qui dura toute la nuit. Au matin, nous avons occupé une tranchée ; il y avait des troupes devant nous; aussi l'officier, laissant un guetteur, nous donna l'autorisation de dormir un peu. Pierre se reposa jusque vers 10 heures. C'est à ce moment que les Allemands commencèrent à paraître sur la crête qui nous faisait face, s'infiltrant un par un, profitant des bouquets d'arbres pour se dissimuler. On nous donna l'ordre de tirer, et bientôt après, une de nos mitrailleuses était repérée et mise hors de service, un homme tué, un autre sérieusement blessé. Pierre essaya de porter, avec quelques camarades, le corps de ce camarade vers l'arrière. Mais bientôt, la ligne qui était devant nous, ayant fléchi, nous recevions l'ordre du repli et devions l'abandonner. Nous gagnons, par un boyau, une tranchée plus à l'arrière où nous essayons d'arrêter la progression ennemie, mais bientôt débordés par la droite, nous devons reculer par bonds successifs, sur le bled, sous un feu intense de mitrailleuses ${ }^{35}$.

La séquence de phrases simples et la suite de marqueurs spatio-temporels contribuent à l'évocation du décès de ce jeune soldat, dont on souligne la bravoure, l'esprit chrétien et la générosité. L'emploi des temps du passé aide à attribuer à cette brève expérience de vie le caractère intemporel et édifiant des histoires exemplaires et le célébrer comme un héros et un martyre de la patrie.

Bien qu'elles aient la modeste prétention de servir de témoignages biographiques aussi, les N3 sont entachées d'exagération rhétorique dans le but de peindre les défunts comme des prototypes de vertus, de dévouement à la famille et à la nation, des esprits cultivés manifestant toujours leur intelligence, finesse, politesse, sensibilité et amour pour les arts et la littérature. Par conséquent,

\footnotetext{
${ }^{34}$ Ibidem.

${ }^{35}$ Le Gafouilleur, n48, 1er juin 1918, p. 1.
} 
on ne transmet que le reflet du personnage tel que la postérité doit le percevoir, en cristallisant l'idéal d'une génération et d'une société. Voici quelques exemples :

[1] Cette mort et cette distinction ne sont point le fait du hasard ; toute l'enfance, toute la jeunesse de Frédéric Charpin l'avaient préparé à ce douloureux triomphe. Ce n'est point que ce grand garçon aux traits énergiques, à la forte moustache, au regard clair, ait eu sur lui cette atmosphère de mélancolie résignée que l'on prête volontiers à ceux qui doivent mourir jeunes. Je le revois encore au Lycée Henri-IV écouter amusé la parole caustique d'Henri Chantavoine; je le revois aux fêtes de Provence, les yeux pleins d'azur, souriant à la beauté des Arlésiennes, au rythme allègre des chansons; je le revois au Luxembourg, sous les marronniers du printemps, où nos causeries amicales mélangeaient, parmi ce jardin italien et français, nos espoirs littéraires et nos regrets méridionaux. Il avait ce courage du sourire, qui sait dissimuler, même aux amis les meilleurs, les soucis et les tristesses, qui par pudeur atténue les grandes pensées, si bien que ses amis eux-mêmes furent étonnés, quand ils virent, à travers ses dernières lettres écrites en août 1914, transparaître toute la beauté de cette âme et ce joyeux et doux lettré prendre tout à coup la figure d'un jeune martyr ${ }^{36}$.

[2] Mais Marcel Bource était aussi, dans le sens le plus large, un artiste. Ses intimes ont seuls vu les pastels élégants et précis qu'il crayonnait, et le public, qui ne recherchait point ignore sans doute encore ses sonnets impeccables ${ }^{37}$.

Dans les N3, les auteurs présentent des éléments qui ont le but d'éclairer les conditions du décès et l'attitude du soldat face à son sort funeste. Pour garantir la véridicité de l'information, le scripteur - qui est d'habitude un camarade du même secteur ou régiment - écrit à la première personne et prétend avoir assisté à ses derniers instants dans le champ de bataille. Le trépas s'entoure alors de son contexte circonstanciel, puisqu'il survient dans une situation exceptionnelle (la guerre) et de manière violente et tragique.

C'est en se relevant pour faire un nouveau bond que Pierre a été frappé. Le voyant tomber, je me suis approché de lui. Je le croyais seulement blessé, il m’a dit : « Je meurs » et a ajouté : «Tu leur diras que j'ai bien aimé le bon Dieu » - puis il m'a demandé de l'embrasser. Quand je l'eus fait, il parut pénétré d'un grand calme et dit simplement : «C'est bien», ses derniers

${ }^{36}$ Bulletin des écrivains de 1914-1915-1916, n 16, février 1916, p. 1.

${ }^{37}$ Bulletin des écrivains de 1914-1915-1916, n 18 , avril 1916, p. 2. 
mots : acte de résignation. Il est mort très doucement, sans se débattre. Nous avons fait notre possible pour emporter son corps; mais, à quelques centaines de mètres de là, j'ai dû l'abandonner [...]. Il n'est presque jamais possible de porter la dépouille de ceux qui tombent. Pierre Baudry avait le pressentiment de mort ; il avait fait le sacrifice de sa vie ${ }^{38}$.

Les N3 incluent enfin un ensemble de données factuelles sur le milieu où le défunt a vécu et agi, sur les grands événements qui ont marqué son existence et sur le rôle qu'il a joué dans les mouvements politiques et intellectuels de son temps. Il est clair qu'on propose une synthèse des faits principaux de sa vie et non pas une chronique comme dans les littératures funéraires classiques. Et cette synthèse est réalisée au moyen de l'hyperbole et du panégyrique, afin d'exalter la figure du Poilu qui s'est immolé pour sauver la France.

[1] Issu d'une famille catholique, professant des opinions de droite, Pierre Baudry, dans la tranchée, donnera le parfait exemple de cette union sacrée qui a toujours régné dans cette petite presse du front où collaboraient côte à côte, fraternellement, des soldats de toutes les classes, de toutes les confessions, de toutes les opinions politiques. [...] En mars 1916 - il avait dixneuf ans - il fonde le Gafouilleur $[\ldots]^{39}$.

[2] Breton aux yeux couleur de mer, Provençal blond dont la voix assourdie tempérait l'accent léger, cet ami fidèle devait à sa double origine de demeurer pour toujours un obstiné rêveur. Comme tant d'autres, il tenta le chimérique accord du rêve et de l'action! Tour à tour marin, journaliste, romancier, puis soldat - soldat de 1914 ! - Lucien Dellys témoigna d'une de ces vertus qu'on nous prêche pour demain et qu'il pratiquait naturellement: celle du parfait $\operatorname{artisan}^{40}$.

\section{Conclusion}

Même si les auteurs se veulent des rapporteurs impartiaux des événements, ils ne peuvent pas s'empêcher de manifester leur engagement, leur intention morale et apologétique, ou encore leurs émotions et leur personnalité. En d'autres mots, pour eux, il n'est pas question de parler d'une mort fictionnelle ou de fictionnaliser la mort d'un personnage historique ; il s'agit plutôt

\footnotetext{
${ }^{38}$ Le Gafouilleur, op. cit.

${ }^{39}$ Ibidem.

${ }^{40}$ Bulletin des écrivains de 1914-1915-1916, n 16, février 1916, p. 1.
} 
de donner une information et, en deuxième lieu, de la transformer en une occasion de mise en valeur de l'ethos des combattants par les biais de l'émotion, du pathos.

Si dans l'Antiquité, la plainte funèbre avait pour but de fixer l'image de ce qu'une société attendait de son élite, en ne célébrant que des héros, rois, hauts prélats, etc., les nécrologies de la Grande Guerre sont l'occasion de rendre hommage aux disparus de n'importe quelle classe sociale. La conséquence directe de cette 'démocratisation' de la mort est la fleuraison, à la fin du conflit, de beaucoup de monuments : dans ces stèles funéraires, les noms des paysans se retrouvent à côté de ceux des docteurs, des notables ou des hommes d'Église et de lettres. De cette manière, un jeune de dix-neuf ans, tel Pierre Baudry, qui fonde un petit journal de tranchées, peut devenir un héros et un modèle pour les générations futures grâce à son sacrifice sur les champs de bataille. C'est ainsi que, comme le soutient Philippe Ariès, «la guerre de 1914 a donné au culte civique des morts «de nos combats mémorables » une diffusion et un prestige qu'il n'avait jamais connus auparavant ${ }^{41}$.

Le défunt devient un miroir des aspirations de la société à la perfection : ses qualités individuelles sont l'expression des lignes de force de la civilisation entière, en conférant à l'éloge personnel une dimension universelle et permanente. Ce n'est pas un hasard si la presse du front développe une rhétorique fondée sur la comparaison antithétique entre Poilus et Boches, entre le véritable esprit gaulois et la prétendue 'Kultur' allemande. Les N2 et N3 se construisent aussi sur une vision assez stéréotypée du deuil, représenté comme l'effet de la force maligne des Allemands, délibérément tendue vers la destruction de la civilisation et de la véritable culture européenne. Au lieu de l'invective contre la mort, typique des genres anciens, on trouve souvent l'invective contre les Boches et leur brutalité, qui fonctionne comme une sorte de catharsis des sentiments profondément ressentis au moment d'un trépas. Quoique la mort soit qualifiée de 'cruelle', 'méchante', 'infâme' et 'arracheuse', elle n'est pas l'ennemi par excellence (ce rôle est par contre attribué au Kaiser et à ses alliés) ou n'est pas vilipendée, grâce à l'intervention de la notion théologique de mors prima qui permet de concevoir un dénouement heureux pour le défunt. La mort glorieuse au champ d'honneur peut donc être considérée comme 'douce' ; pensée qui doit consoler les vivants, atténuer leur douleur et vaincre la crainte de l'oubli par l'assurance du souvenir éternel.

${ }^{41}$ Philippe ARIÈS, L'Homme devant la mort, Paris, Seuil, coll. « L’univers historique », 1977, p. 543. 